\title{
Post-Electrophoretic Identification of Oxidized Proteins
}

\author{
Craig C. Conrad, John M. Talent, Christina A. Malakowsky, and Robert W. Gracy* \\ Molecular Aging Unit, Department of Molecular Biology and Immunology, University of North Texas \\ Health Science Center, Fort Worth, Texas, 76107
}

"To whom correspondence should be addressed: Robert W. Gracy Ph.D., Office of Research and Biotechnology, ME-2 Room 806, 3500 Camp Bowie Blvd, Fort Worth, Texas 76107, Fax (817) 735-5485; E-mail: rgracy@hsc.unt.edu

Abbreviations used: DNPH, 2,4-dinitrophenylhydrazine; PVDF, polyvinyldienefluoride; DNPantibody, dinitrophenyl-antibody; SDS, sodium dodecyl sulfate; ROS, reactive oxygen species.

\begin{abstract}
The oxidative modification of proteins has been shown to play a major role in a number of human diseases. However, the ability to identify specific proteins that are most susceptible to oxidative modifications is difficult. Separation of proteins using polyacrylamide gel electrophoresis (PAGE) offers the analytical potential for the recovery, amino acid sequencing, and identification of thousands of individual proteins from cells and tissues. We have developed a method to allow underivatized proteins to be electroblotted onto PVDF membranes before derivatization and staining. Since both the protein and oxidation proteins are quantifiable, the specific oxidation index of each protein can be determined. The optimal sequence and conditions for the staining process are (a) electrophoresis, (b) electroblotting onto PVDF membranes, (c) derivatization of carbonyls with 2,4-DNP, (d) immunostaining with anti DNP antibody, and (e) protein staining with colloidal gold.
\end{abstract}

\section{INTRODUCTION}

Humans are continually exposed to reactive oxygen species (ROS) that are produced in tissues from normal metabolism or the inflammatory response of leukocytes or macrophages (1), (2). Due to these unavoidable insults, it has been estimated that as much as $1 \%$ of the consumed oxygen may be converted to ROS (3), which may cause damage to cellular components such as DNA, lipids and proteins. Oxidative modifications to proteins can lead to cross-linking, peptide fragmentation, modified residues, and the conversion of one amino acid to another (4). If sufficient protein damage accumulates, cell death will occur. Although several antioxidant defense systems have evolved to prevent ROS damage, oxidized proteins appear to accumulate with age and may represent $30-50 \%$ of the total protein in old cells (3).

The oxidative modification of proteins has been shown to play a major role in a number of human diseases. The ability to identify specific proteins that are most susceptible to oxidative modifications is

(C) 2000 Biological Procedures Online. All rights reserved. Paper-based copying permitted for internal use for educational or non-profit purposes only. Otherwise, this article may be copied to paper provided that \$US15 per copy is paid directly to Biological Procedures Online. Electronic copying, storage or redistribution prohibited. 
key to the development of methods for early diagnosis, assessment of new potential therapies and understanding the overall disease mechanisms. Therefore, it is important to have reliable methods to identify specific oxidized proteins. We have recently described (5) an analytical procedure that allows both the reversible staining of total proteins and the specific immunostaining of the oxidized proteins separated on polyacrylamide gels. However, a shortcoming of the procedure is that it requires the protein mixture (e.g., cell or tissue extract) be pre-derivatized prior to electrophoretic separation. For example, the protein derivatization of carbonyl functional groups with 2,4-dinitrophenylhydrazine (DNPH) is used for antibody recognition of oxidized proteins. Unfortunately, this pre-derivatization alters the electrophoretic (and electrofocusing) properties of proteins. Consequently, it is not possible to directly compare the patterns from "oxidized" fingerprints with those from "non-oxidized" protein fingerprints that have been compiled into large databases. The ability to conduct all derivatization and staining after electrophoresis and transblotting would offer many additional advantages over prederivatization procedures (e.g. determining oxidation of samples that have been blotted for prior experiments). Thus, the goal of this study was to develop a method that would permit direct comparison of the peptide fingerprint database of normal proteins and, at the same time, reveal the proteins that are preferentially subject to oxidative damage on a single Western blot. The method was also designed to allow determination of the specific oxidation index (i.e. nanomoles of carbonyl per $\mathrm{mg}$ of protein) of any oxidized protein that was not derivatized prior to Western blotting.

\section{MATERIALS AND METHODS}

\section{Electrophoresis and Transblotting}

Samples were mixed with Laemmli Sample Buffers and separated electrophoretically on a $10 \%$ acrylamide gel $(16 \mathrm{~cm} \times 18 \mathrm{~cm}$ plates) (Hoefer/LKB). The gels were then equilibrated in electrotransfer buffer (25mM Tris, 191mM Glycine, 15\% methanol) and electroblotted to either nitrocellulose or

PVDF membranes. Both single dimensional and two-dimensional separations were utilized and details were described previously (5) or as indicated in the legends to the figures.

\section{Derivatization}

Following the electroblotting procedure, the PVDF membrane (Millipore) was immersed in 100\% methanol $(15 \mathrm{sec})$, and then dried at room temperature. The Nitrocellulose membranes were removed from the blotting apparatus and dried completely at room temperature. Prior to derivatization, both membranes were equilibrated in $20 \%$ (v/v) methanol-80\% (v/v) TBS for 5 minutes. Continuous shaking was used during all incubation and washing steps. Membranes were incubated in $2 \mathrm{~N} \mathrm{HCl}$ for 5 minutes. The membranes were next incubated in a solution of 2,4-dinitrophenylhydrazine $(0.5 \mathrm{mM})$ in $2 \mathrm{~N} \mathrm{HCl}$ for exactly 5 minutes each, as described by Robinson et al. (6). The membranes were washed three times in $2 \mathrm{~N} \mathrm{HCl}$ (5 minutes each) and five times in 100\% methanol (PVDF) or 50\% methanol (nitrocellulose), for 5 minutes each wash.

\section{Total Protein Staining}

CPTS Reversible Stain. The method used for total protein staining using Copper [II] $\underline{P h t h a l o c y a n i n e}$ 3,4,4',4" 'TetraSulfonic acid tetrasodium salt (CPTS) was a modification of the method described by Bickar and Reid (7). Membranes were made acidic by incubation in $12 \mathrm{mM} \mathrm{HCl}$ for 10 minutes. The 
membranes were then incubated for 10 minutes (or until blue bands appeared) in a $0.05 \%$ (w/v) solution of CPTS (5). To remove excess stain, the membranes were rinsed 3 times with $12 \mathrm{mM} \mathrm{HCl}$ (for 5 minutes each wash). The proteins on the membrane were visualized using a CCD digital camera, as described below. To remove the CPTS stain, the membranes were washed twice with $0.5 \mathrm{M}$ sodium bicarbonate for 5 minutes each wash, then washed with water until bands were no longer visible (approximately 30 minutes).

SYPRO Rose Reversible Protein Blot Stain (8). The manufacturer's protocols were used for total protein staining using SYPRO Rose protein blot stain (Molecular Probes, Eugene, OR). Washing and staining steps were performed with continuous mechanical agitation. Membranes were immersed in SYPRO Rose Blot Wash solution and incubated at room temperature for 15 minutes in a small polypropylene staining dish. This wash step was repeated with both membranes. The membranes were then rinsed four times with water (10 minutes each wash). Membranes were completely immersed in SYPRO Rose Blot Stain and allowed to incubate for 15 minutes. The excess dye was removed by rinsing 4-6 times (1 minute per wash) in deionized water. Membranes were visualized using UV or 488 nm light. SYPRO Rose stain was removed prior to subsequent derivatization or immunostaining procedures. Immersing the membranes in SYPRO Rose Blot Destain solution for 15 minutes with gentle agitation at room temperature completely destained the membranes. Membranes were then washed two times with deionized water for 1 minute

SYPRO Ruby Protein Blot Stain (9). The manufacturer's protocols were used for total protein staining using SYPRO Ruby protein stain (Molecular Probes, Eugene, OR). Washing and staining steps were performed with continuous mechanical agitation. The transblotted proteins were fixed to the nitrocellulose and PVDF by completely immersing the membrane in a 7\%(v/v) acetic acid: $10 \%(\mathrm{v} / \mathrm{v})$ methanol solution and incubating at room temperature for 15 minutes in a small polypropylene staining dish. The membranes were washed four times with water (for five minutes each wash). Membranes were then completely immersed in SYPRO Ruby Blot Stain Reagent and allowed to incubate for 15 minutes. The excess dye was removed by rinsing 2-3 times in deionized water. Because the SYPRO Ruby stain is a permanent protein stain and only visible during illumination with UV or $488 \mathrm{~nm}$ light it was not removed prior to subsequent derivatization or immunostaining.

Colloidal Gold (10). The manufacturer's protocols were used for total protein staining using Colloidal Gold protein stain (BioRad, Hercules, CA). Washing and staining steps were performed with continuous mechanical agitation. The membranes were washed three times with TBS-Tween (10 minutes each wash), and then rinsed 3 times for 2 minutes with deionized water. Membranes were then completely immersed in Colloidal Gold and incubated until bands reached the desired intensity (approximately 1 hour). The excess dye was removed by rinsing 3 times for approximately one minute in deionized water. Membranes were allowed to dry before visualization. The Colloidal Gold stain is a permanent protein stain and was not removed prior to subsequent derivatization or immunostaining.

Immunostaining. The PVDF membranes were immunostained using a modification of the protocol described by Mansfield (1995) (11). Membranes were incubated for 1 hour at $25^{\circ} \mathrm{C}$ with the primary antibody solution consisting of a 1:16,000 dilution of the rabbit anti-2-4-dinitrophenol antibody (Molecular Probes) in PBS-Tween containing 5\% milk. The membranes were then washed three times with PBS-Tween solution for two minutes each, and incubated with a 1:2,500 (v/v) dilution of the goat 
-anti-rabbit (BRP-conjugated) secondary antibody (Sigma) in PBS-Tween containing 5\% milk (w/v) for 1 hour at room temperature. The membranes were then washed 3 times for 2 minutes each in PBS-Tween solution prior to visualization.

The nitrocellulose membranes were incubated for 5 minutes in PBS-Tween. To block the nonspecific protein binding sites the membranes were incubated with 5\% milk (w/v) in PBS-Tween for 1 hour. The membranes were washed once for 5 minutes in PBS Tween. Membranes were then incubated for 1 hour at $25^{\circ} \mathrm{C}$ with the primary antibody solution A) consisting of a 1:16,000 dilution of the rabbit anti2-4-dinitrophenol antibody (Molecular Probes) in PBS-Tween containing 5\% milk as described previously (5). The membranes were washed three times with PBS-Tween solution for 10 minutes each, and incubated with a 1:2,500(v/v) dilution of the goat anti-rabbit (BRP-conjugated) secondary antibody (Sigma) in PBS-Tween containing 5\% milk (w/v) for 1 hour at room temperature. The membranes were washed 3 times for 10 minutes each in PBS-Tween solution prior to visualization

\section{Visualization and quantification of images}

A computerized cooled CCD camera-based imaging system (Alpha Innotech) was used to visualize and record the stained proteins. For CPTS, and Colloidal Gold stained material, the membranes were illuminated from above using an epi-white fluorescent light source. For the SYPRO Ruby and SYPRO Rose protein blot stains, the membranes were illuminated from above and below using an epi-UV-B $(\sim 300 \mathrm{~nm})$ light source with a $490 \mathrm{~nm}$ long-pass filter. A chemiluminescence kit (SuperSignal $^{\circledR}$ West $^{2}$ Femto Maximum Sensitivity Substrate, Pierce) was used to visualize the immunostained blots. Following exposure to the chemiluminescent chemicals, the membranes were placed in a light-tight cabinet and a cooled CCD camera captured the light produced by the chemical reaction with the enzyme that is linked to the secondary antibody. The CCD camera shutter remained open for several minutes to capture the light produced. Protein bands were quantified using AlphaEase ${ }^{\mathrm{TM}}$ densitometry software (Alpha Innotech) and expressed as the Integrated Density Values (IDV).

\section{RESULTS AND DISCUSSION}

The goal of this study was to develop and optimize a method that would allow mixtures of proteins to be first separated electrophoretically then stained both for total protein and oxidized protein. Moreover, the objective was to obtain quantitative measurements of total protein and oxidized protein on single bands of protein from a single transblotted gel, thereby permitting determination of the "specific oxidation index" for any protein.

Shacter et al (12) developed a method for derivatized mixtures of proteins with 2,4-DNP, and separated them using Poly Acrylamide Gel Electrophoresis (PAGE). The proteins were then electroblotted to nitrocellulose and immunostained with an anti-DNP antibody. Using this method it is possible to observe individual proteins that have been oxidized. However, this method did not allow a means of visualizing the specific protein content of individual bands on the same blot, and thus determination of the specific level of oxidation of each protein relied upon a "duplicate" gel and blot. Therefore, it was desirable to develop a method for measuring both the specific protein content and specific oxidation level of individual proteins directly on membranes following electrophoresis and electroblotting. 
Figure 1A shows a total protein stain of a polyacrylamide gel with increasing concentrations of BSA. The electrophoretic separation and total protein stain allows individual proteins to be quantitated (e.g. arrows Figure 1A). Following derivatization with 2,4-DNP and immunostaining with anti-DNP antibody, a linear increase in signal intensity was observed (Figure 1B). Using these methods, the ratio of oxidation to total protein can be calculated and the specific oxidation index (representing the nanomoles of oxidation per mg of protein) can be determined for each individual protein of the resolved mixture.

The goal was to optimize conditions that would allow complex mixtures of both specific proteins and specific oxidized proteins to be visualized using same membrane. The first optimization was to determine what support matrix (PVDF or nitrocellulose) was better suited for these types of analyses. Next was to determine which commercially available protein stain would yield the best resolution of total proteins. Finally, to determine if the order of staining and derivatization would affect quantitation of the results.

Figure 2 shows protein profiles of human sera electroblotted on two different membranes (Nitrocellulose and PVDF). Figure 2A shows proteins that were visualized with four different stains; Figure 2B shows the proteins visualized after the derivatization with DNPH; and Figure 2C shows the proteins visualized after both the derivatization and immunostaining. Figure 2A shows that proteins electroblotted to nitrocellulose and PVDF had different affinities for the four protein stains. For example, when membranes were visualized with the reversible CPTS stain, the proteins transferred to PVDF did not visualize

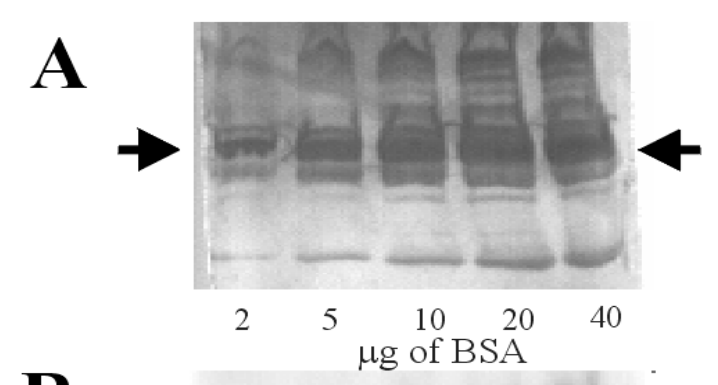

B
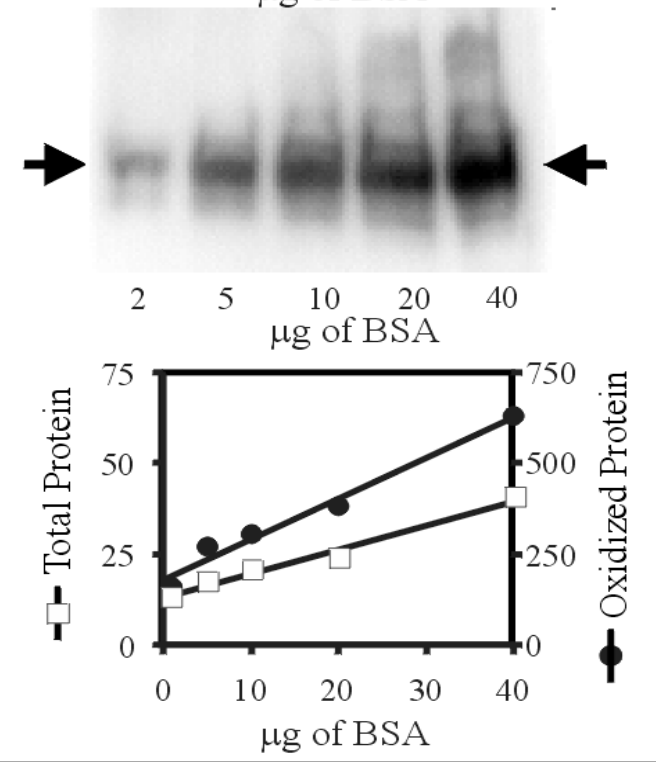

Fig 1. PAGE quantification of total and oxidized bovine serum albumin (BSA). Panel A represents a total protein stain of different concentrations of BSA $(2,5,10,20,40 \mu \mathrm{g}$ of BSA). Panel B represents the oxidized proteins of different concentrations of BSA $(2,5,10,20,40 \mu \mathrm{g}$ of BSA) immuno-stained with 2,4-DNP antibody. Panel C shows the linear increase in proteins and oxidation levels of the large band (arrows) of BSA shown in Panel A \& B. Filled circles

$(\bullet)$ represent oxidized protein, and open squares represent ( $\square$ ) total protein. These values were measured in Integrated Density Values (IDV) as described in Materials and Methods. as well as the proteins bound to nitrocellulose (Figure 2A-CPTS). In contrast, when the membranes were stained with the reversible SYPRO-Rose, the proteins on nitrocellulose did not stain as well as those blotted onto the PVDF (Figure 2A-SYPRO Rose). The same proteins, when visualized using the non-reversible colloidal gold stain showed equivalent staining patterns regardless of which membranes were used (Figure 2A-Colloidal Gold). Similarly, proteins stained with the non-reversible SYPRORuby stain showed equal staining patterns, regardless of what membrane was used for blotting. 
Colloidal gold staining was superior in terms of the sensitivity and was essentially identical on both types of membranes.

Next, it was necessary to determine whether the derivatization with DNPH would affect the different stains used to visualize the transblotted proteins. These data are compared in Figure $2 \mathrm{~B}$. The derivatization process markedly interfered with the subsequent staining of the proteins with SYPRO Rose (Figure 2B-SYPRO Rose) on either type of membrane. The intensity of the SYPRO Ruby stain was diminished on both types of membranes following derivatization, however, PVDF appeared to be more affected (Figure 2B-SYPRO Ruby). The colloidal gold protein staining patterns that resulted after the derivatization protocol (Figure 2B-Gold), were essentially the same as the protein patterns observed when the proteins were stained without derivatization (Figure 2A-Gold). Moreover, the two types of membranes were equally suitable.

Finally, Figure $2 \mathrm{C}$ shows the total protein staining patterns for the protein stains after the membranes were first derivatized, immunostained and then stained for total protein. The patterns were essentially identical to those seen in the Figure 2B series in which the membranes were only derivatized prior to the protein stain. Again, the colloidal gold procedure was far superior in terms of sensitivity no matter what type of membrane was used. These data also show that neither the immunoblotting, nor the chemiluminescence chemicals interfere with the colloidal gold total protein stain.

In order to determine whether the different protein stains would interfere (or enhance) the ability of DNP-antibody to detect oxidized proteins, we conducted a series of

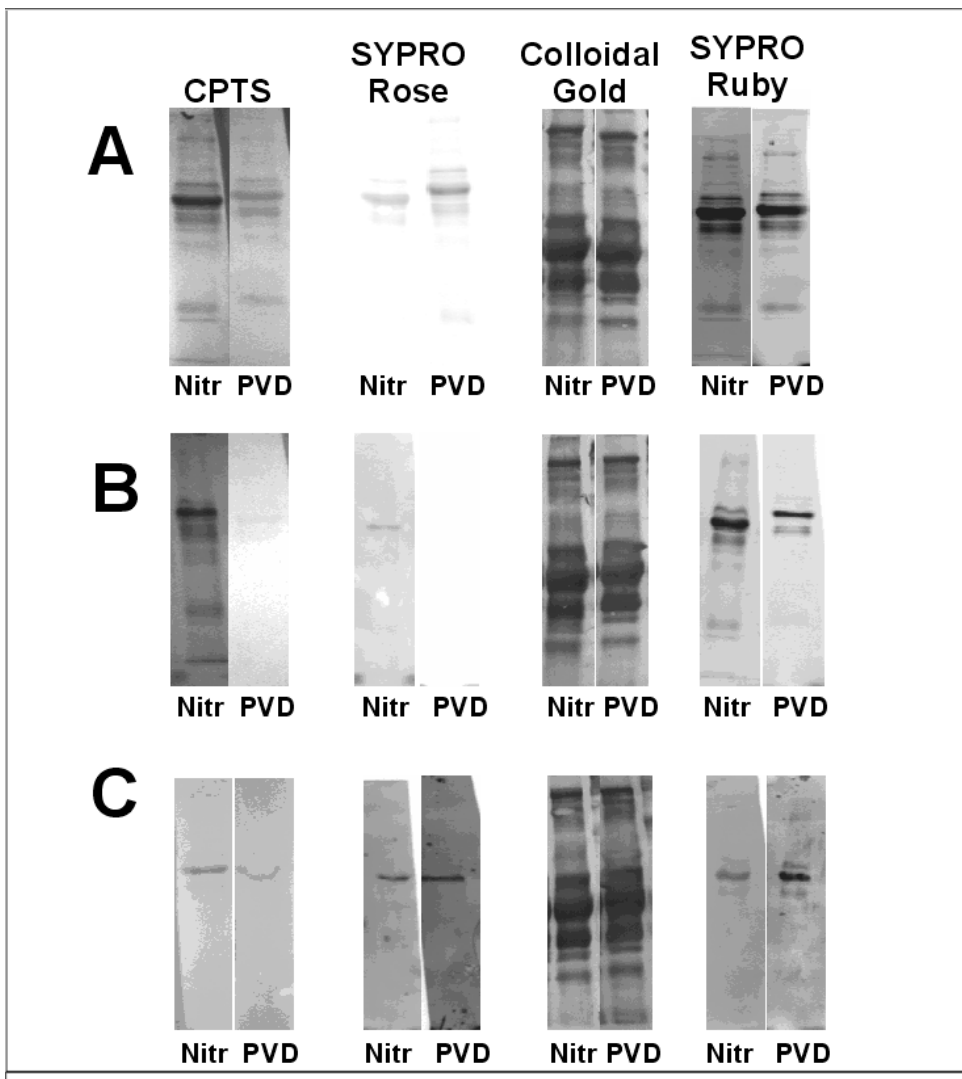

FIG 2. Visualization of total membrane bound proteins with different protein stains. Human blood serum from a single donor was used for all blots. The replicate samples were prepared, separated, and analyzed using one-dimensional PAGE according to protocols described by Talent, et al (5). Proteins were electroblotted to either Nitrocellulose (Nitr), or PVDF (PVD) as described in Materials and Methods. The blots were cut into strips and total proteins were stained with; CPTS, SYPRO Rose Ruby Red, Colloidal Gold, or SYPRO Ruby as indicated. Panel (A) represents the protein blots that were stained directly after electroblotting. Panel (B) represents protein blots that were first derivatized with DNPH, as described in material and methods, and then stained for total protein. Panel (C) represents protein blots that were first derivatized with DNPH, then immunostained using anti-2, 4-DNP, then proteins stained using the indicated stain. experiments and quantified the intensity of the chemiluminescent immunostaining signal. Each total protein stain that was used prior to derivatization showed increased chemiluminescent signals, which indicated the various total protein stains were reacting with the derivatization solutions (data not shown). Thus, we concluded protein staining prior to derivatization yielded unnecessary artifacts. 
Following the derivatization step and subsequent washing steps, the PVDF membrane had a bleach white appearance. In contrast, the nitrocellulose membranes had a faint yellow appearance. This uptake of DNP in the nitrocellulose membrane presumably increased the background signal caused by antiDNP antibody, which decreased chemiluminescent signal intensity for the nitrocellulose membrane. Thus, the PVDF membranes yield a stronger chemiluminescence signals when compared to nitrocellulose membranes. A further advantage of PVDF membranes over nitrocellulose is that if the sample is to be subjected to microsequence analysis, the PVDF membrane can be added directly to the sequencer.

The most pronounced limitation of the above technique is that Western blotting is a relatively imperfect quantitative technique. There are quantitative limitations inherent in any immunochemical blot assay. For example, variations in epitopes can affect the intensity of staining or derivatization, and chemiluminescence exposures times are known to vary from blot to blot. However, because Western blots are so sensitive, this limitation is typically accepted.

From the above studies we have concluded the following: First, post-electrophoretic identification of oxidized proteins using 2,4-DNP is possible; Secondly, post-electrophoretic identification of specifically oxidized proteins can be quantitative and the specific oxidation index can be determined; Thirdly, colloidal gold is preferred as the protein stain. This is due to not only its superior sensitivity but also the lack of interference with the immunostaining. Fourth, PVDF membranes are preferred for immunoblotting; and Fifth, the optimal sequence for the staining process is (a) electrophoresis, (b) electroblotting, (c) derivatization with DNPH, (d) immunostaining, and (e) protein staining with colloidal gold.

\section{ACKNOWLEDGMENTS}

This work was supported in part from grants from the R.A. Welch Foundation (BK0502) and the Alzheimer's Association.

\section{REFERENCES}

1. Cadenas, E., Boveris, A., Ragan, C. I., and Stoppani, A. O. 1977. Archives of Biochemistry \& Biophysics 248-257.

2. Wakeyama, H., Takeshige, K., Takayanagi, R., and Minakami, S. 1982. Biochem J 593-601.

3. Stadtman, E. R. 1988. J.Gerontol. B112-B120.

4. Davies, K. J. 1987. J.Biol.Chem. 9895-9901.

5. Talent, J. M., Kong, Y., and Gracy, R. W. 1998. Anal.Biochem. 31-38.

6. Robinson, C. E., Keshavarzian, A., Pasco, D. S., Frommel, T. O., Winship, D. H., and Holmes, E. W. 1999. Anal.Biochem. 48-57.

7. Bickar, D. and Reid, P. D. 1992. Anal.Biochem.109-115.

8. Lim, M. J., Patton, W. F., Lopez, M. F., Spofford, K. H., Shojaee, N., and Shepro, D. 1997. Anal.Biochem. 184-195.

9. Neumann, H. and Mullner, S. 1998. Electrophoresis 752-757.

10. Rohringer, R. and Holden, D. W. 1985. Anal.Biochem. 118-127.

11. Mansfield, M. A. 1995. Anal.Biochem. 140-143.

12. Shacter, E., Williams, J. A., Lim, M., and Levine, R. L. 1994. Free Radic.Biol.Med. 429-437 